\title{
Tiefes Leid und enorme Großzügigkeit
}

\section{Einsichten eines Informatikers von geringem Verstande}

\section{Reinhard Wilhelm ${ }^{1}$}

Online publiziert: 6 . Oktober 2020

๑) Springer-Verlag GmbH Deutschland, ein Teil von Springer Nature 2020

Das Internet ist ja in der letzten Zeit eher durch die Verbreitung von Hass und Lügen aus höchstem Munde in Verruf gekommen. Was dabei ganz untergeht, ist die Großzügigkeit, die sich täglich in unseren Mailboxen manifestiert. Die meisten Äußerungen enormer Großmut werden uns aber aus falsch verstandener Fürsorge von unseren Providern erst gar nicht zur Kenntnis gebracht. Aber es kommen noch genug durch, um unser Herz wegen der auf uns persönlich gezielten Aufmerksamkeit zu erfreuen. Allerdings gibt es Verbesserungsmöglichkeiten, die unsere oft ein wenig eingetrübte Freude zu einer vollkommenen machen würden.

Eine Anrede mit Hallo \{Vorname\} \{Nachname\} und exklusiver Information, bestimmt für: $\{$ Email $\}$ hat doch etwas Unpersönliches an sich. Man fühlt sich nicht richtig persönlich äschtimiert, wie der Saarländer sagt.

Das ist schon anders, wenn die Familie von Kirk Douglas mir Gutes tun möchte. Da fühle ich mich schon sehr geehrt! Dass die Familie dieses alten Zausels ausgerechnet an mich denkt.

Sehr geehrter Begünstigter

Wir hoffen, dass diese Nachricht Sie gut findet. Na klar, hat mich ja gefunden, ob jetzt gut oder schlecht, ist ja wurscht.

Wir senden zufällig Nachrichten an Personen, um zu sehen, ob sie einen Teil des Vermögens der Hollywood-Legende Kirk Douglas erhalten möchten. Obwohl ein Teil seines Vermögens für wohltätige Zwecke bestimmt war, finden wir (sein Nachlass) Ihre E-Mail als eine davon. Ich sehe ein, dass ich nicht die optimale Zielperson für Wohltätigkeit bin. Umso netter, dass sie an mich denken.

Reinhard Wilhelm

wilhelm@cs.uni-saarland.de

1 Saarland Informatics Campus, Saarbrücken, Deutschland
Mit freundlichen Grüßen, der Nachlass von Kirk Douglas. Ja, Nachlass, großzügiger Zeitgenosse! Ist wirklich nett von dir! Ich mache mit!

Es bricht eine ganze Welle großzügigster Spendenbereitschaft über mir zusammen.

Erstaunlich, wer sich alles meldet. Sie wissen offensichtlich nicht, dass mein Verlag mich für meine Glossen fürstlich entlohnt.

Frau Paul aus dem Elfenbein möchte mich reich machen: Es ist mir eine Freude, Ihnen nach dem Wiegen zu schreiben, weil Sie nicht von Angesicht zu Angesicht sehen können. Offensichtlich freut sich Frau Paul, dass sie ordentlich etwas auf die Waage bringt, was ich leider nicht sehen kann, weil sie ihrer E-Mail kein Bild angefügt hat.

Was mich immer wieder rührt ist die Kombination von tiefem Leid und riesiger Großzügigkeit.

Frau Janet Jackson Anderson schreibt mir mit schweren Tränen in ihren Augen und großer Trauer in ihrem Herzen, dass sie wegen eines Krebsproblems nicht für den Zeitraum von sieben Monaten durchhalten würde. Das würde eigentlich schon reichen, um mich zu motivieren, ihre großzügige Spende anzunehmen. Aber es kommt sogar noch ein Schlaganfall hinzu! Sie möchte, dass ich $30 \%$ von 45.850.000,00 USD für meinen persönlichen Gebrauch verwende, ... Das Geld hat ihr der verstorbene Ehemann hinterlassen. Die beiden waren elf Jahre lang ohne Ehefrau verheiratet Kind. Sie schreibt, „Ich bin als Waisenkind aufgewachsen und habe keinen Körper als mein Familienmitglied, ... Tue dies, damit Gott meine Sünden vergibt und meine Seele akzeptiert, weil diese Krankheiten mich so sehr gelitten haben."

Man kommt leicht auf die Idee, dass Gott ihr wohl für ihre sprachlichen Sünden eine verdiente Strafe hat zukommen lassen.

Dennis Jefferson wurde mit Kehlkopfkrebs diagnostiziert und möchte deshalb alle seine Ressourcen wohltäti- 
gen Zwecken zur Verfügung stellen. Da ist er bei mir an der richtigen Adresse!

Der Vater von Miss Bintou Deme wurde vergiftet und hat ihr ein riesiges, legal erworbenes Vermögen hinterlassen, welches sie mir teilweise übereignen möchte. Da kann man ja schon mal helfen!

Frau Muna Skaf wurde von einer Gasattacke in Syrien erwischt und will mir großzügiger Weise $15 \%$ von US\$4.500.000,00 schenken.

Shane Missler möchte mir 2.000.000,00€ spenden. Einfach so! Aber, ohne dass sie zumindest einen sauberen Krebs hat, eine Gasattacke in Syrien erlitten hat oder ohne Ehemann verheiratet war, mache ich nicht mal meine Hosentasche für das Geld auf.

Mein Name ist Charles W. Jackson Jr. ... Sie haben sich für Spenden-/Humanitäre Mittel in Höhe von 3 Mio. gewonnen. EUR besteht, um den Ausbruch von COVID-19 in Ihrer Region zu gehören, zu einer globalen Katastrophe wurde, bevor er die Wirtschaft lähmt. Ehrlich gesagt, bin ich jetzt etwas verwirrt, ob diese Mittel für mich oder gegen Covid19 sind.

Erstaunlich ist auch, wie viele Lotteriegewinner mich an ihren Gewinnen teilhaben lassen wollen! Ich dachte immer, die Teilnehmer an Lotterien wollten selbst reich werden, und finde es deshalb rührend, wie selbstlos sie sind.

Mein Name ist Manuel Franco aus den USA. Ich habe die America-Lotterie im Wert von 768Mio. US-Dollar ge- wonnen und spende einen Teil davon an nur 5 glückliche Menschen und einige Waisenhäuser als Wohlwollen für die Menschheit, und ein paar Tage später: Manuel Franco Powerball Winner has donated $\$ 4.500 .000$ to you.

Auch eine Frau Lerynne West kümmert sich gleich mehrfach rührend um mich: Hallo, ich bin Lerynne West, Sie haben eine Spende von $€ 1.930 .000,00$. Ich habe am 5. November 2018 die 343,9Mio. Powerball-Lotterie gewonnen. Ich spende einen Teil davon an zehn glückliche Menschen und die Ten Charity-Organisation. Ihre E-Mail ging als Sieger hervor. Wenden Sie sich dringend an die Schadenabteilung.

Sie möchte mir beim nächsten Mal eine Spende von 2.800.000,00€ zukommen lassen, ,ich gewann die Amerika-Lotterie im Wert von 343 Mio. Dollar und ich spende einen Teil davon an funf gluckliche Menschen und Wohltatigkeitshauser."

Wenn man schon 343 Mio. US\$ gewinnt, hätte man nicht bei den Umlauten sparen sollen. Andererseits überrascht den halbwegs mit Statistik vertrauten, dass Frau West und Herr Franco offensichtlich immer gewinnen, wenn sie Lotterie spielen, und Frau West jedes Mal rund 343 Mio. Dollar. Da sollte man wohl die Statistikbücher umschreiben!

Es bleibt festzustellen, dass das Internet und seine Bewohner viel besser als ihr Ruf sind. 